\title{
CANTORI, ISLANDS AND ASYMPTOTIC CURVES IN THE
}

\section{STICKINESS REGION}

\author{
C. EFTHYMIOPOULOS ${ }^{1,2}$, G. CONTOPOULOS ${ }^{1,2}$ and N. VOGLIS ${ }^{2}$ \\ ${ }^{1}$ Research Center for Astronomy, Academy of Athens \\ ${ }^{2}$ Department of Astronomy, University of Athens
}

\begin{abstract}
The resonant structure near a noble cantorus is found. Islands of stability are located near the gaps of the cantorus. The crossing of the gaps of the cantorus by the asymptotic curves of unstable periodic orbits is shown numerically (non-schematically). We discuss how these structures influence stickiness.
\end{abstract}

\section{Introduction}

The phenomenon of "stickiness" occurs when a chaotic orbit starting near a last KAM torus remains close to this torus for long times before diffusing into a surrounding large chaotic sea. Among the first to give examples of stickiness were Contopoulos (1971), and Shirts and Reinhardt (1982).

A theoretical understanding of stickiness was advanced mainly for 2D systems (e.g. Greene 1979, MacKay 1983, MacKay et al. 1984; see Meiss 1992 for a review). It is now established that stickiness is due to the existence of cantori (Aubry 1978, Percival 1979, Mather 1982, Schmidt and Bialek 1982).

Cantori are the remnants of the KAM tori formed when the latter are destroyed by an increase of the perturbation. A cantorus forms a Cantor set of points on the surface of section. This set is invariant under the section mapping and has rotation number equal to the rotation number of its progenitor KAM curve.

A cantorus constitutes a partial barrier to local chaotic diffusion. Chaotic orbits can cross the cantorus only by passing through the gaps of the cantorus.

On the other hand, the global chaotic transport is limited by the existence of several consecutive cantori in the stickiness region. It is numerically observed that in many cases the holes of such cantori are delineated along "chimneys" (MacKay et al. 1984), where chaotic transport mainly takes place.

MacKay (1983) found numerically that the flux through gaps is minimal for those cantori having noble rotation numbers, i.e. numbers of which the continued fraction

$$
a=\left[a_{1}, a_{2}, \ldots\right] \equiv 1 /\left(a_{1}+\left(1 / a_{2}+\ldots\right)\right)
$$

(where $a_{i}$ are integers) has $a_{i}=1$ for all $i$ above a certain order $N$. Thus, only noble cantori are important for the stickiness problem.

MacKay et al. (1984) modelled the flux through a gap of the cantorus as a "turnstile" and obtained estimates of transport rates by assuming a simple Markov chain model with given transition probabilities between any two successive cantori.

In order to construct a more accurate model of diffusion through cantori, one requires knowledge of the resonant phase space structure in the stickiness region. 


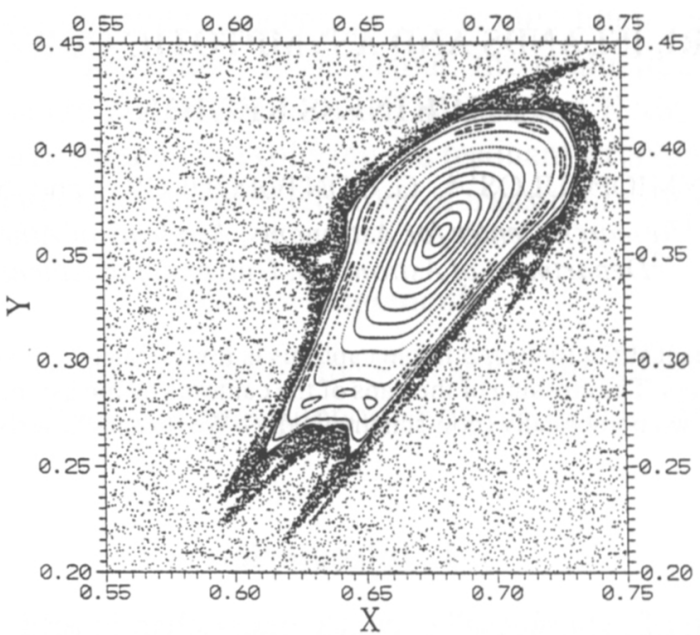

Fig. 1. The main island of stability and the surrounding stickiness region in the standard map for $K=5$.

In particular, we find that the chaotic diffusion through a cantorus is substantially influenced by: a) the existence of islands of stability close to the gaps of a cantorus, and $b$ ) the form of the asymptotic curves (of unstable periodic orbits) crossing the gaps of the cantorus.

In this paper we summarize some of our results based on a numerical study of the standard map and some new results that give a better understanding of the stickiness problem. The earlier results are presented in greater detail elsewhere (Efthymiopoulos et al. 1997, Contopoulos et al. 1998).

\section{The Stickiness Zone}

We take as our model the $2 \mathrm{D}$ standard map

$$
x_{i+1}=x_{i}+y_{i+1}, \quad y_{i+1}=y_{i}+\frac{K}{2 \pi} \sin \left(2 \pi x_{i}\right) \quad(\bmod 1)
$$

where $K$ is the nonlinearity parameter. When $K=5$ the phase space (unit square) is mostly chaotic, but contains two symmetric islands of stability around the stable periodic orbit $x_{c} \approx 0.68, y_{c} \approx 0.36$ of period 2 . Considering only every second iteration, the orbit $\left(x_{c}, y_{c}\right)$ can be considered of period 1 . The island around this stable periodic orbit is shown in Fig. 1. This island is surrounded by a stickiness zone consisting of two parts. An inner thin zone (very dark) where the stickiness time is large, of order $10^{4}-10^{7}$ periods, and a more extended outer zone (less dark), where the stickiness time is smaller, of order $10^{2}$. Outside the stickiness region is the large chaotic sea. 


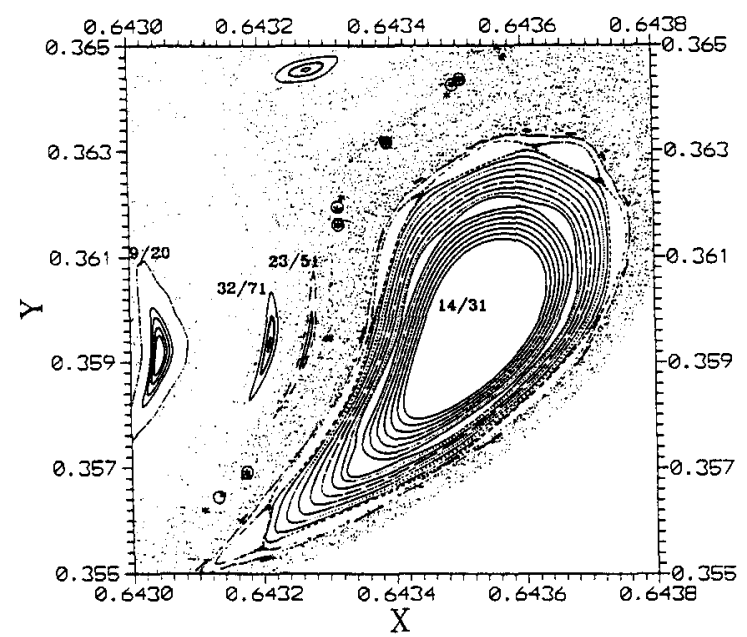

Fig. 2. A detail of the phase space near the border of the main island for $K=5$. We distinguish the islands of stability $9 / 20,14 / 31$, etc. and the unstable orbits $60 / 133$ (triangles), $97 / 215$ (squares) $157 / 348$ (circles) and $254 / 563$ (stars).

\section{Cantorus and Islands}

The most important cantorus in the stickiness region of Fig. 1 is the one with the noble rotation number $[2,4,1,1, \ldots]$. This cantorus is formed at the critical value $K_{c} \approx 4.9974$ and surrounds the main island of stability.

The successive rational truncations of the noble number $[2,4,1,1, \ldots]$ are:

$$
\frac{1}{2}, \frac{4}{9}, \frac{5}{11}, \frac{9}{20}, \frac{14}{31}, \frac{23}{51}, \frac{37}{82}, \frac{60}{133}, \frac{97}{215}, \frac{157}{348}, \frac{254}{563}, \frac{411}{911} \ldots
$$

and they form a Farey sequence. These rationals correspond to periodic orbits surrounding the main island, one inside (closer to the island) and one outside the cantorus. They approach the cantorus closer as the order increases.

Fig. 2 shows a detail of the phase space where several periodic orbits are marked. In particular, the periodic orbits $9 / 20,14 / 31$ and $23 / 51$ are stable and are surrounded by islands (the island $32 / 71$ does not belong to the sequence (3)), while the periodic orbits $97 / 215,254 / 563$ (inside the cantorus), and $60 / 133,157 / 348$ (outside the cantorus) are unstable. These unstable orbits are very close to each other and they define essentially the gaps of the cantorus.

In Fig. 2, the island $23 / 51$ is outside but very close to a cantorus gap. Such islands close to the gaps pose limits to chaotic diffusion.

The existence of islands close to the gaps, for $K$ slightly above $K_{c}$, is guaranteed by the fact that the critical value $K_{c}$ is the minimum of the critical values of destabilisation of the periodic orbits (3) (Contopoulos et al. 1987).

For every rational $n / m$ of the sequence (3) correspond two periodic orbits, one stable and one unstable, which bifurcate from the central periodic orbit (of period 


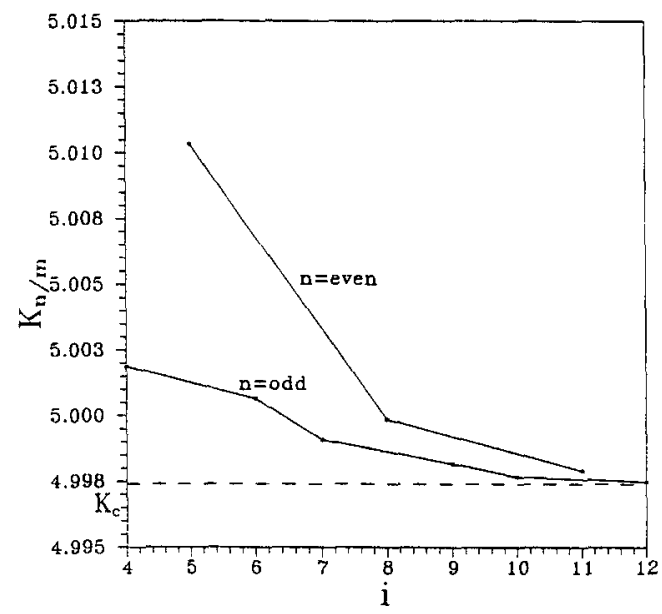

Fig. 3. The critical value $K_{n / m}$ of destabilisation of the orbit $n / m$ versus its corresponding order $i$ in the sequence (3).

1) at particular values of the perturbation $K$. As $K$ increases, both orbits move from the center outwards. At some particular perturbation value $K_{n / m}$, the stable periodic orbit $n / m$ becomes unstable by a single or double period bifurcation.

According to Greene's criterion (Greene 1979), the series of the critical values $K_{n / m}$ of the successive orbits $n / m$ has as its limit the critical value $K_{c}$ at which the noble torus $[2,4,1,1, .$.$] turns to a cantorus.$

In Fig. 3 we give the critical values $K_{n / m}$ versus the order of the orbits in the sequence (3). As the order increases, the values $K_{n / m}$ converge to a limit $K_{c}=4.9974 \ldots$ (horizontal line). The convergence follows two different lines, when the numerator $n$ is even (upper line) or odd (lower line). Thus the deviations of $K_{n / m}$ from the limiting value $K_{c}$ are larger for $n=$ even than for $n=$ odd (for the same order of $n$ ). The value $K_{c}=4.9974$.. is the critical value of destruction of the noble torus $[2,4,1,1, \ldots]$. It is clear that the critical value $K_{c}$ is the minimum of the critical values $K_{n / m}$. Thus, for any particular orbit $n / m$, there is a finite interval of values $K_{c}<K<K_{n / m}$ for which the orbit is stable while the corresponding cantorus is already formed. Such stable orbits are surrounded by islands which limit diffusion through the gaps of the cantorus.

In Figs.4a,b the evolution of Hénon's stability index $a_{n / m}$ is given for each of the initially stable orbits $n / m$ of the sequence (3). For all orbits the stability index starts from $a_{n / m}=1$ at the perturbation value $K$ of their generation from the center. As $K$ increases, the stability index $a_{n / m}$ initially decreases slowly and later abruptly. Then there are two possibilities: a) the stability index $a_{n / m}$ crosses the line $a=-1$ at the critical perturbation $K_{n / m}$ where the orbit becomes unstable. This happens when the numerator $n$ of the periodic orbit is odd. b) the stability curve $a_{n / m}$ becomes tangent to the line $a=-1$ and is reflected. Then the orbit becomes unstable at the value $K_{n / m}$ when $a_{n / m}$ crosses the line $a=+1$. This 

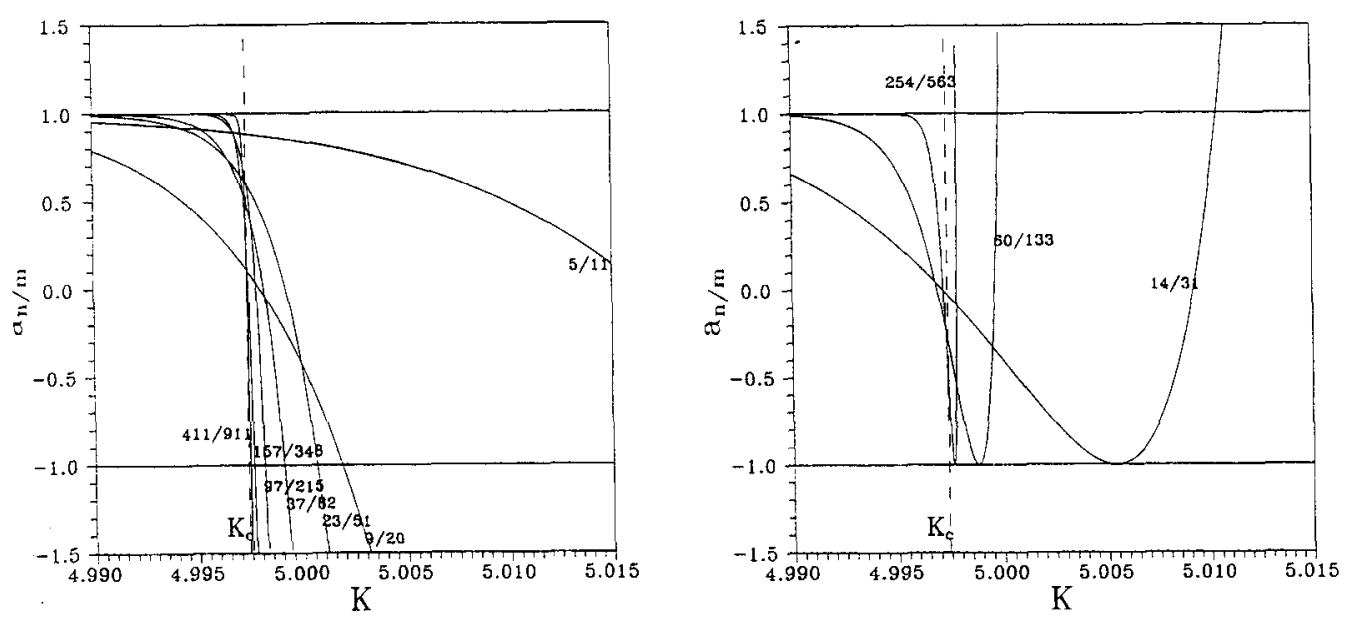

Fig. 4. The Henon stability index as a function of $K$ for the orbits of the sequence (3). The vertical line is at the critical value $K_{c}$ of destabilisation of the noble torus $[2,4,1,1, \ldots]$. a) Orbits with $n=$ odd. b) Orbits with $n=$ even.

happens when the numerator $n$ is even.

In any case, the transition to instability is more abrupt as the order of the periodic orbit increases. In Fig.4a, we show the evolution of the stability index of the orbits with odd numerator $5 / 11,9 / 20,23 / 51,37 / 82,97 / 215,157 / 348$, and $411 / 911$ as a function of the perturbation $K$. The stability index curves, near destabilisation, are more abrupt for the higher order periodic orbits. The same phenomenon occurs for the periodic orbits with even numerator 14/31,60/133, and 254/563 (Fig.4b). At the limit of infinite order (cantorus), the stability index curve takes the form of a step function changing slope from zero to $\infty$ abruptly at $K=K_{c}$.

The resonant structure near the noble torus or cantorus can be shown very efficiently by use of the recent method of angular dynamical spectra (Voglis and Efthymiopoulos 1998). Starting from the main center (period-1 orbit), we define the position vector $R_{i}$ of any point of an orbit around the island, and the infinitesimal deviation vector $\xi_{i}$ found by solving the variational equations of the map (2). Then we define the rotation angle $\theta_{i}$ as the angle between two successive vectors $R_{i}$ and $R_{i+1}$, and the twist angle $\phi_{i}$ as the angle between two successive vectors $\xi_{i}$ and $\xi_{i+1}$. Both angles are defined in appropriate intervals (see Voglis and Efthymiopoulos 1998 for details). Finally, we define the distributions of the rotation angles and twist angles (angular dynamical spectra $S(\theta)$ and $S(\phi)$ ). The angular moments are defined as:

$$
\nu_{\theta}=\frac{1}{2 \pi} \oint \theta S(\theta) d \theta, \quad \nu_{\phi}=\frac{1}{2 \pi} \oint \phi S(\phi) d \phi \quad,
$$

and they correspond to the angular frequencies of rotation of the vectors $R$ and $\xi$. 


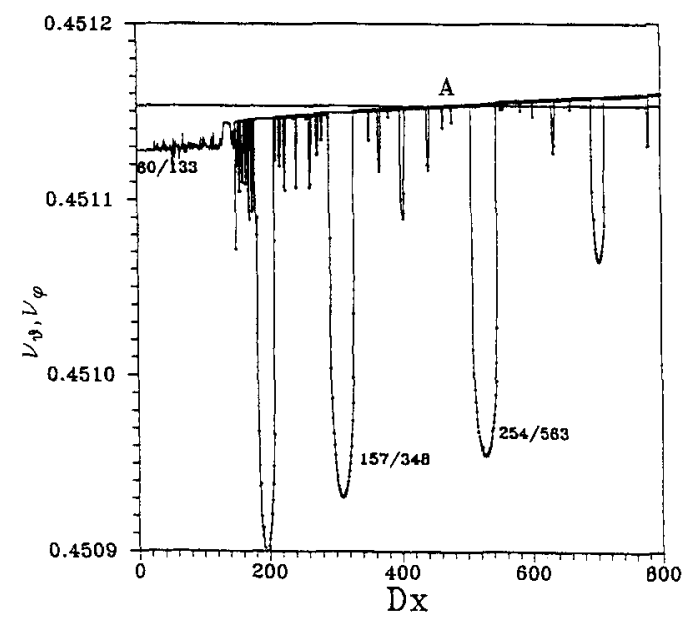

Fig. 5. The curves $\nu_{\theta}(x)$ (solid) and $\nu_{\phi}(x)$ (solid with dots) for $K=4.997$ ( $D x$ is given in units of $3.75 \times 10^{-9}$, starting from $x=0.64364$ ). The point $\mathrm{A}$ where the two curves coincide marks the position of the noble torus $[2,4,1,1, \ldots]$.

Then the following relations hold (Voglis and Efthymiopoulos 1998):

$$
\nu_{\theta}=\nu_{\phi}
$$

for a closed KAM curve and

$$
\nu_{\phi}=\nu_{\theta}-\nu_{k}
$$

for higher order islands of stability, where $\nu_{k}$ is the mean angular frequency of epicyclic motion around the local center (stable periodic orbit) of the island.

In Fig. 5 we calculate the curves $\nu_{\theta}(x)$ and $\nu_{\phi}(x)$ for $K=4.997<K_{c}$. The U-shaped parts of the curve $\nu_{\phi}$ correspond to islands of stablility marked with their rotation numbers. The point $\mathrm{A}$, where both curves $\nu_{\theta}(x)$ and $\nu_{\phi}(x)$ coincide on the horizontal line $\nu_{\theta}=\nu_{\phi}=[2,4,1,1, .$.$] , gives the position of the corresponding$ noble torus. The curves $\nu_{\theta}(x)$ and $\nu_{\phi}(x)$ coincide in a small segment around $A$, indicating that the noble torus $[2,4,1,1, .$.$\} and nearby tori still exist. The$ coincidence of the curves $\nu_{\theta}(x)$ and $\nu_{\phi}(x)$ in a segment around $[2,4,1,1, .$.$] means$ that the volume of tori in the very close neighborhood of the noble torus tends to unity. This can be understood by a recent theorem of Morbidelli \& Giorgilli (1995). Further away we distinguish many islands of stability. The islands $60 / 133$, $157 / 348$ and $254 / 563$ belong to the sequence (3), while the rest of the islands belong to other Farey sequences.

The size of the islands for $K=4.997$, as a function of their multiplicity, is given in Fig. 6. We see that the islands with $n=$ even are larger than those of the same order with $n=$ odd. The sizes of the islands for $n=$ even and $n=$ odd follow two parallel lines that correspond to the power law

$$
D S=A m^{-2.75}
$$




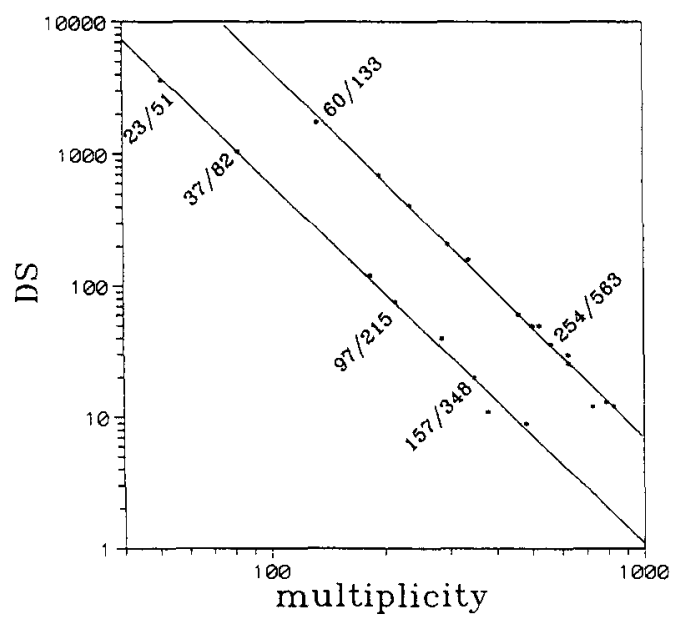

Fig. 6. The size of the islands as a function of the multiplicity $m$ for $K=4.997$. The sizes $D S$ are measured along a line joining the periodic orbits $14 / 31(x=0.6438327$, $y=0.3593945)$ and $23 / 51(x=0.6437657, y=0.3631837)$, and are given in units of $1.267 \times 10^{-7}$. The sizes for $n=$ even and $n=$ odd follow the power law (7) with different constants $A$. The islands that belong to the Farey tree (3) are marked.

with $A=2.8 \times 10^{8}$ for $n=$ odd and $A=1.7 \times 10^{9}$ for $n=$ even.

When $K$ grows beyond $K_{c}$ the higher order periodic orbits become unstable and the corresponding islands are replaced by chaotic regions. This can be seen in Fig. 7 that gives the values of $\nu_{\theta}(x)$ and $\nu_{\phi}(x)$ for $K=4.9975>K_{c}$. In this case the noble torus $[2,4,1,1, \ldots]$ has been destroyed and transformed into a cantorus. Then, the diference $\nu_{\theta}-\nu_{\phi}$ is no longer equal to zero, but it is minimum near the cantorus (Voglis et al. 1998). In Fig. 7 we see still some islands of lower order around the cantorus. These islands act as partial barriers for the diffusion of the orbits, and contribute to the stickiness in their neighbourhood. Even for $K=5$ the low order orbits $4 / 9,5 / 11,9 / 20,14 / 31$ and $23 / 51$ are still stable. Some of the corresponding islands of stability are shown in Fig. 2.

\section{Stickiness and Asymptotic Curves}

The asymptotic curves of unstable periodic orbits inside a cantorus cross the gaps of the cantorus and act also as barriers to chaotic diffusion. A numerical (nonschematic) example of such crossing of the cantorus by an ustable asymptotic curve is given in Fig. 8. The unstable asymptotic curve starting at the point $O$ of the unstable periodic orbit $87 / 215$ (inside the cantorus $[2,4,1,1, .$.$] ) is plotted$ until the time when it crosses the cantorus for the first time (Fig.8). This happens after 4 iterations of an initial segment of size $\Delta S_{0}=10^{-10}$ along the asymptotic curve near the unstable periodic orbit. The unstable asymptotic curve first moves downwards and makes four oscilations inside the cantorus. Then it moves upwards 


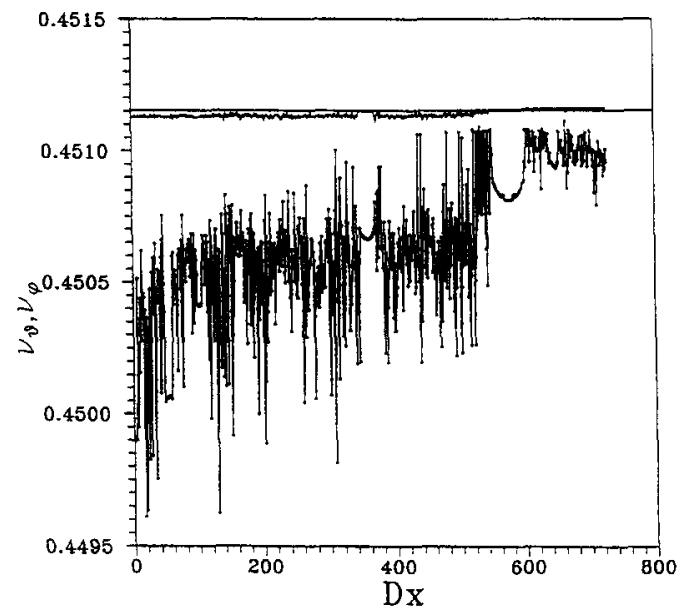

Fig. 7. Same as Fig. 5 but for $K=4.9975$ ( $D x$ starts at $x=0.643583$ ). The two curves do not coincide anywhere, i.e. the noble torus no longer exists.

and crosses a gap of the cantorus. After its exit from the gap, the asymptotic curve moves almost parallel to the cantorus. If we iterate for longer times, we find that the asymptotic curve makes several oscillations crossing the cantorus successively inwards and outwards, before going to the large chaotic sea outside the island. The size of the asymptotic curve is approximately $\Delta S=\Delta S_{0}|\lambda|^{n}$ at the nth iteration, where $\lambda$ is the larger eigenvalue of the periodic orbit. In the present case $\lambda \approx-190$ and the escape to the large chaotic sea occurs after $n=6$ iterations.

The unstable asymptotic curve of Fig. 8 moves for some time almost parallel to the cantorus because the islands of stability close to the gaps of the cantorus (e.g. the islands 23/51) do not allow large excursions of the asymptotic curve in the transverse direction to the gaps. Furthermore, the asymptotic curve itself acts as a partial barrier to chaotic diffusion, since it cannot be intersected by the unstable asymptotic curve of any other unstable periodic orbit further inside the cantorus. Thus, for the latter to cross a gap of the cantorus, it necessarely has to move following a parallel path to the one of the unstable asymptotic curve of Fig. 8.

We conclude that the diffusion of any chaotic orbit inside the cantorus follows essentially the same path as defined by the unstable asymptotic curves which emanate from unstable periodic orbits inside the cantorus.

If we decrease the value of $K$ the stickiness time increases and becomes infinite when $K$ is smaller than the critical value $K_{c}$. In fact, as $\mathrm{K}$ decreases, the sizes of the gaps of the cantorus decrease and become zero for $K=K_{c}$. However the decrease of the size of the gaps is not the only reason for the increase of the stickiness time. In Fig. 9 we show part of the asymptotic curve of the unstable periodic orbit 254/563 which starts inside the cantorus $[2,4,1,1, \ldots]$ for $K=4.998$. This asymptotic curve passes through the gaps of the cantorus and finally reaches the large chaotic 


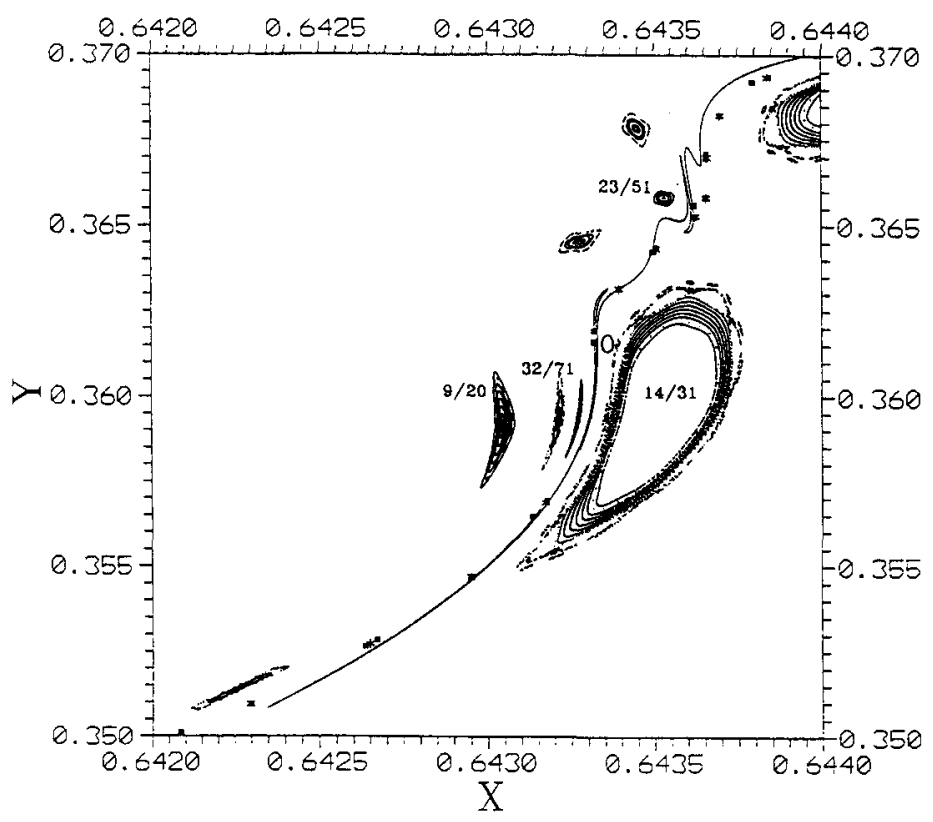

Fig. 8. Crossing of the cantorus $[2,4,1,1, \ldots]$ by the unstable asymptotic curve of the orbit $97 / 215$ for $K=5$. The stars and the squares represent the periodic orbits $97 / 215$ (inside the cantorus) and $157 / 348$ (outside the cantorus), and they define approximately the gaps of the cantorus.

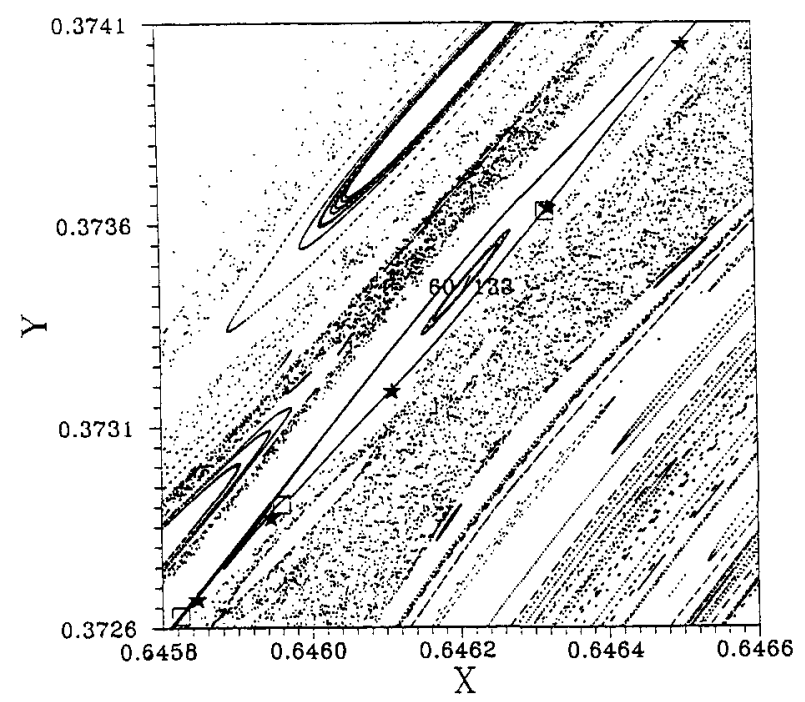

Fig. 9. The structure of the phase space near the cantorus $[2,4,1,1, \ldots]$ for $K=4.998$. The cantorus is approximated by the periodic orbits $157 / 348$ (stars, outside the cantorus) and 254/563 (squares, inside the cantorus). A part of the asymptotic curve of the periodic orbit $254 / 563$ is also plotted (solid line), corresponding to the 11th iteration of an initial segment $\Delta S_{0}=10^{-10}$. 
sea.

In this case the eigenvalue of the periodic orbit is about $\lambda \approx 8$. The first crossing of the cantorus occurs at the 9 th iteration (starting with an initial segment $\Delta S_{0}=10^{-10}$ ). This is only a little over double the time required for crossing the same cantorus in the case $K=5$. However for larger times the behaviour is very different. In Fig. 9 we see that after 11 iterations the asumptotic curve surrounds the islands $60 / 133$ outside the cantorus, but there is no indication of a further diffusion outwards. In fact, we have found that an orbit requires a much longer time (of the order of $10^{7}$ iterations) in order to reach the outer chaotic sea.

We conclude:

(a) that the stickiness is much more pronounced in the case $K=4.998$ than in the case $K=5$. This is related to the fact that $K=4.998$ is quite close to the limiting value $K_{c}=4.9974$,

(b) that the stickiness is not due only to the cantorus $[2,4,1,1, \ldots]$. Other cantori, outside the island $60 / 133$, plus the higher order islands in this region produce an important barrier for the diffusion of the orbits in this neighboorhood. A further study of this phenomenon is in progress.

\section{Acknowledgements}

This research was supported in part by the Research Committee of the Academy of Athens (grant 200/409). C.E. was supported by the Greek Foundation of State Scholarships (IKY)

\section{References}

Aubry, S.: 1978, in Bishop, A.R. and Schneider, T.(eds) "Solitons and Condensted Matter Physics", Springer, p.264.

Contopoulos, G.: 1971, Astron.J., 76, 147.

Contopoulos, G., Varvoglis, H. and Barbanis, B.: 1987, AstronAstrophys., 172, 55.

Contopoulos, G., Harsoula, M., Voglis, N., and Dvorak, R.: 1998, "Destruction of Islands of Stability", (in preparation).

Efthymiopoulos, C., Contopoulos, G., Voglis, N., and Dvorak, R.: 1997, J. Phys. A:, 30, 8167.

Greene, J.M.: 1979, J.Math.Phys., 20, 1183.

MacKay, R.S.: 1983, Physica, 7D, 283.

MacKay, R.S., Meiss J.D., and Percival I.C.: 1984, Physica, 13D, 55.

Mather, J.N.: 1982, Topology, 21, 457.

Meiss, J.D.: 1992, Rev.Mod.Phys., 64, 795.

Morbidelli, A., and Giorgilli, A.: 1995, J. Stat. Phys., 78, 1607.

Percival, I.C.: 1979, in Month, M. and Herrera, J.C. (eds) "Nonlinear Dynamics and the Beam-Beam Interaction", Amer.Inst.Phys. p.302.

Schmidt, G., and Bialek, J.: 1982, Physica, 5D, 397.

Shirts, R.B. and Reinhardt, W.P.: 1982, J.Chem.Phys, 77, 5204.

Voglis, N., and Efthymiopoulos, C.: 1998, J. Phys. A, 31, 2913.

Voglis, N., Contopoulos G., and Efthymiopoulos, C.: 1998, this volume. 